\title{
Verteilt arbeiten, gemeinsam gewinnen
}

\section{Christof Ebert}

Eingegangen: 22. Januar 2021 / Angenommen: 4. Juni 2021 / Online publiziert: 5. Juli 2021

(C) Springer Fachmedien Wiesbaden GmbH, ein Teil von Springer Nature 2021

Zusammenfassung Verteiltes Arbeiten muss heute jeder beherrschen. Aktuell zeigt die Covid-19 Pandemie die Vorteile verteilter Arbeit: Effizienz, Flexibilität, geringere Kosten und Overheads sowie weniger Reisen und damit ökologisches Verhalten. Doch viele Unternehmen erreichen nicht, was sie wollen. Im Homeoffice geht die Produktivität um ein Drittel zurück. Die Hälfte der Sourcing-Projekte wird vor Abschluss abgebrochen. Dieser Beitrag gibt aus der Praxis einen Überblick zu den Chancen und Risiken von verteiltem Arbeiten. Basierend auf einer weltweiten Umfrage mit IEEE Software werden Trends nach der Covid-19 Pandemie sowie erste Erfahrungen vertieft. Ein erstes Fazit: Verteiltes Arbeiten ist eine massive Transformation. Bauen Sie die dafür nötigen Kompetenzen schrittweise aus: Kultur, Soft Skills, Prozesse und viel Geduld.

Schlüsselwörter Kollaboration · Zusammenarbeit · Führung · Virtuelle Teams · Kompetenzmanagement

Folgen Sie ihm auf Twitter: @ ChristofEbert.

Christof Ebert $(\square)$

Ingersheimerstr. 24, 70499 Stuttgart, Deutschland

E-Mail: christofebert@ieee.org 


\title{
Work distributed, win together
}

\begin{abstract}
Today, everyone must master distributed work. Currently the Covid-19 pandemic shows the advantages of distributed work: efficiency, flexibility, lower costs, less overhead and less travel and thus ecologic behavior. Yet many companies are not achieving their desires. Productivity drops by a third in the home office. Half of sourcing projects are abandoned before completion. This article provides a practical overview of the opportunities and risks of distributed working. Based on a worldwide survey with IEEE Software, trends following the Covid-19 pandemic and initial experiences are explored in depth. An initial conclusion: distributed working is a massive transformation. Build the skills needed for it step by step: Culture, soft skills, processes, and lots of patience.
\end{abstract}

Keywords Collaboration · Cooperation · Leadership · Virtual teams · Competence management

\section{Verteilt arbeiten, gemeinsam gewinnen}

Verteiltes Arbeiten muss heute jeder beherrschen. Nicht zuletzt die Corona-Pandemie zeigte die Vorteile verteilter Arbeit: Effizienz, Flexibilität, geringere Kosten, weniger Overheads und nicht zuletzt weniger Reisen und damit ökologisches Verhalten. Doch viele Unternehmen erreichen nicht, was sie wollen. Im Homeoffice geht die Produktivität um ein Drittel zurück. Die Hälfte der Sourcing-Projekte wird vor Abschluss abgebrochen. Dieser Beitrag gibt aus der Praxis einen Überblick zu den Chancen und Risiken von verteiltem Arbeiten. Basierend auf einer weltweiten Umfrage mit IEEE Software werden Trends nach der Covid-19 Pandemie sowie erste Erfahrungen vertieft. Ein erstes Fazit: Verteiltes Arbeiten ist eine massive Transformation. Bauen Sie die dafür nötigen Kompetenzen schrittweise aus: Kultur, Soft Skills, Prozesse und viel Geduld.

\section{Motivation: Warum verteilte Teams?}

„Across the Great Wall we can reach every corner in the world" war der Text der ersten E-Mail, die jemals aus China gesandt wurde. Dies war am 20. Sep. 1987, und interessanterweise ging diese erste chinesische E-Mail nach Deutschland, an die Universität Karlsruhe. Dieser bescheidene Satz, der es in seiner Tragweite leicht mit demjenigen von Neil Armstrong anlässlich des ersten Schritts eines Menschen auf dem Mond aufnehmen kann, drückt das gesamte Spannungsfeld verteilten Arbeitens aus. Verteiltes Arbeiten kennt keine Grenzen, hier bildhaft dargestellt mit der Chinesischen Mauer. Insbesondere Dienstleistungen rund um IT, Produktentwicklung, aber auch in der Lehre oder Forschung kennen weder Landesgrenzen noch physikalische Limits und lassen sich über die ganze Erde verbreiten. Die kurze Zeitdauer von dieser Internet-Steinzeit bis heute zeigt, wie dynamisch und praktisch nicht vorhersehbar die Entwicklung der Informationstechnik wirklich ist. 
Abb. 1 Motivation für verteiltes Arbeiten: Flexibilität, Innovation und Effizienz
3. Effizienz Wettbewerbsfähigkeit, Kosten, Exzellente Prozesse, Geschwindigkeit
1. Flexibilität Skalierung, Zugriff auf aktuell nötige Kompetenzen, Just-In-Time Netzwerke, Ökosysteme

2. Innovation Neue Ideen, Globale Strategie für Wachstum, Zugang zu lokalen Märkten und Ideen

Diese erste Mail, aus China zeigt natürlich auch die Risiken im Zusammenhang mit verteiltem Arbeiten. Viren erreichen ebenfalls jede Ecke der Welt, wie die Corona-Pandemie zeigte. Der Wettbewerbsdruck und häufig verzerrte Marktbedingungen und versperrte Marktzugänge, wie wir sie auch in China beobachten, erschweren das verteilte Arbeiten. Schließlich haben wir (Selbst-) Ausbeutung, wie beispielsweise in der Gig-Economy, und Lohn-Dumping als große Herausforderungen, denen man auf regionaler Ebene und selbst politisch kaum begegnen kann - es sei denn man stoppt den freien Dienstleistungsverkehr.

Warum also verteiltes Arbeiten? Die wesentlichen Treiber sind schnell benannt: Flexibilität, Innovation und Effizienz. Abb. 1 zeigt den Zusammenhang dieser Faktoren (Ebert 2020). Für die meisten Unternehmen steht die Flexibilität im Vordergrund. Vorbei sind die Zeiten, wo es ausschließlich um Kostenreduzierung ging. Heute ist klar, dass erfolgreiche verteilte Geschäftsmodelle alle drei Dimensionen berücksichtigen müssen (Ebert 2020). Daher unterscheiden wir auch klar zwischen Kosten und Effizienz. Mehr Effizienz bringt in der Regel Kostenreduzierung - aber nicht notwendigerweise, wenn die Mittel investiert werden. Effizienz hat eine sehr viel größere Tragweite, beispielsweise, wenn man schnell Arbeitsinhalte zwischen Standorten verlagern kann, oder mit Kunden rasch an einem Standort zusammenarbeiten kann. Dieser Effekt, der natürlich auch zu einer Kostenreduzierung beiträgt hat sich mit Distanzarbeit nochmals verstärkt. Das gilt auch für Freelancer, die mit dem Übergang zum ,neuen Normal“ mit mehr Online-Arbeit die Auslastung sehr viel besser optimieren können, denkt man beispielsweise an Alleinerziehende, die damit in freien Zeitblöcken arbeiten können. Wir wollen in diesem Beitrag allerdings die Perspektive der Unternehmen beibehalten.

\section{Erfahrungen in der Covid-19 Pandemie}

Inmitten dieser globalen Verwerfungen hat Vector gemeinsam mit IEEE Software weltweit Experten in Technologieunternehmen gebeten, ihre Meinung zu den Herausforderungen der Industrie sowie Erfahrungen aus der Covid-19 Pandemie darzustellen. Dazu haben wir Entscheidungsträger in Unternehmen im weltweiten B2B- 
Abb. 2 Industrie-Trends 2021 Innovation in stürmischen Zeiten



Kontext zu den größten Herausforderungen befragt, denen sie sich kurzfristig und mittelfristig stellen müssen. Die Umfrage zeigt eine gute Validität mit 3,5\% Rücklaufquote von 2700 Empfängern aus verschiedenen Branchen weltweit. Diese Rückläufer hatten den Fragebogen in allen Fällen komplett ausgefüllt.

Abb. 2 zeigt die Ergebnisse der aktuellen Umfrage zu Industrietrends vom Jahresende 2020. Die horizontale Achse zeigt kurzfristige Herausforderungen, und die vertikale Achse zeigt mittelfristige Herausforderungen. Jeder Teilnehmer hatte maximal fünf Stimmen, so dass die Summe der Datenpunkte bei über $100 \%$ liegt. Zusätzlich haben wir mit Unternehmen gesprochen, um zu vertiefen, wohin sich Technologien entwickeln und welche Themen kurz- und mittelfristig relevant sind. Wir können in der Abbildung drei Cluster gut unterscheiden. Ins Auge sticht der Datenpunkt rechts oben mit Innovation. Das ist eine sowohl kurzfristige wie auch langfristige Herausforderung für praktisch alle Unternehmen, wie die Umfrage zeigt. Das Cluster rechts zeigt den kurzfristigen Druck im weltweiten Wettbewerb, wie wir ihn bereits über viele Jahre sehen. Er ist in der Wahrnehmung zurückgegangen im Vergleich zur Vorgängerumfrage, was das positive Klima hinsichtlich Innovation und Aufbruch zeigt. Das Feld in der Mitte mit Themen wie Flexibilität und Komplexitätsbeherrschung ist wichtig, wird aber als weniger dringend angesehen. Klar ist, dass Innovation einige dieser Themen subsummiert, beispielswese Digitale Transformation. Das verteilte Arbeiten wanderte nach unten, da es im neuen Normal angekommen ist. Da gibt es kein Zurück, und man kann damit umgehen.

Ende 2019, also im ,,alten Normal“" vor Covid-19, waren viele Unternehmen bereits in wirtschaftlich schwieriger Situation und sahen als wesentliche kurzfristige Herausforderung das Überleben in der Krise (Ebert 2020; Stelter 2020). Damals war das Cluster mit Qualität, Kosten, Kompetenzen rechts oben. Dieser Fokus führt unweigerlich in einen Teufelskreis von Verdrängungswettbewerb oder „Red Ocean“. Kostenreduzierung und Sparmaßnahmen ohne passende Kompetenzen führen zu Qualitätsprobleme, die den Kostendruck nochmals beschleunigen. Eine typische Abwärtsspirale, wie wir sie von vielen Unternehmen kennen, die sich in der Krise kaputtsparen. Reflexartiges Verhalten in der Krise, wie reine Kostensenkungsprogramme, ist gefährlich, denn in der Regel wird an Innovation und Qualität gespart. 
Ein einfaches Beispiel ist die Streichung von Mitarbeiter-Schulungen, um Kosten zu sparen. Diese wiederum sind demotiviert und haben nicht die nötige Energie und das Know-how, um die Krise zu meistern. Das haben wir 2019/2020 oft gesehen, vor allem in Europa und den USA, wo viele Mitarbeiter aufgefordert wurden, weniger zu arbeiten. Da der Arbeitsinhalt gleichblieb, nahmen Verzögerungen und Nacharbeit dramatisch zu. Das bedeutet mehr Kosten, was wiederum zu mehr Kostendruck führt. Ein Teufelskreis, weswegen wir in der Abb. 2 auch einen Blitz zeigen, um die Gefahr dieses kurzsichtigen Verhaltens zu verdeutlichen. Hier kommen wir auf den Unterschied zwischen Effizienz und Kosten zurück. Kosteneinsparungen können helfen, aber sind in der Regel kontraproduktiv. Effizienzsteigerung dagegen schafft nachhaltige Verbesserungen.

2020 stimulierte die Pandemie mit ihren vielen ad-hoc Lockdown Maßnahmen, die wie Flickenteppiche ausgebreitet und ständig revidiert wurden, einen radikalen Paradigmenwechsel. Unternehmen verloren von jetzt auf nachher die Kontrolle über ihre Geschäftsmodelle, Mitarbeiter und Kundenbeziehungen. Die oftmals planwirtschaftlich anmutenden Einschränkungen trafen offensichtlich den Einzelhandel, Kulturschaffende und die Gastronomie besonders. Aber auch die meisten anderen Unternehmen mussten sich in kurzer Zeit neu erfinden. Selbst Krankenhäuser standen vor der Situation, dass nur noch einige wenige Maßnahmen gefordert wurden. Mit einem Schlag wurde branchenübergreifend klar, dass ,weiter so“ mit traditionellen Sparmaßnahmen nichts bringt. Geschäftsmodelle mussten rasch überarbeitet werden. Mit Digitalisierung und KI waren ganz neue Formen der Kundennähe und des Service möglich, wie die Beispiel Chat-Bots im Finanzbereich, Robotic Process Automation (RPA) in der Produktion, und prädiktive Wartung bei Produkten und Anlagen zeigen (Ebert 2020; Ebert und Duarte 2018; McKinsey 2021).

Die neue Normalität verlangt nach Innovation. Selbstgefälligkeit und bloßes Jammern führen ins Abseits. Die Pandemie ist sicher nicht die Ursache der aktuellen Wirtschaftskrise, denn alle Indikatoren waren auch bereits 2019 greifbar. Im Gegenteil: Die Pandemie beschleunigt Veränderung und führt unsere Wirtschaft und Verwaltungen endlich in die Neuzeit. Der weltweite Markt beschleunigt diese Entwicklung. Covid-19-Impfstoffe werden einige Symptome dieser Transformation abschwächen, aber sie werden veraltete Verhaltensweisen nicht heilen. Innovationen und die kontinuierliche Verbesserung unserer Verhaltensweisen sind das wesentliche Instrument, um wettbewerbsfähig zu bleiben. Branchenübergreifend braucht es optimistische Innovationen, um der Abwärtsspirale zu entkommen.

Die Technologietrends sind branchenübergreifend identisch: ACES (Autonomie, Convergence, Ecology, Services). Traditionell trieb Software den Wandel an. In der neuen Normalität treibt der Wandel die Software. Covid-19 hat die Agenda von praktisch jedem von uns verändert (Ebert 2020). Und es hat zahlreiche Innovationen in Software und IT angeregt. Viele Unternehmen waren im vergangenen Jahrzehnt selbstgefällig geworden und gingen davon aus, dass das Wachstum sich einfach fortsetzt. Doch bereits 2019 war die Notwendigkeit von Veränderungen erkennbar, die sich mit Covid-19 dann massiv beschleunigte. Gelegentlich brauchen wir einen Weckruf, um innovativ zu sein. Der hat uns nun erreicht.

Die Arbeitsorganisation ändert sich schnell. Abb. 3 zeigt aus der gleichen Umfrage, wie die Unternehmen die Krise wahrgenommen haben - und welche Muster 
There is much more online collaboration than face-to-face meetings

Digital transformation is a key topic for engineering We need to survive and thus focus on core
business

We focus more on innovation to prepare for tomorrow

Same work needs to be done with less effort

Much stronger cost competition than before

Others

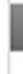



$86 \%$

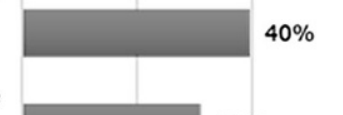

$\%$

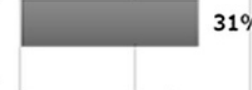

$1 \%$

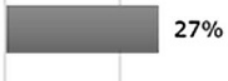

$27 \%$

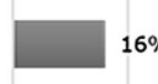

$16 \%$

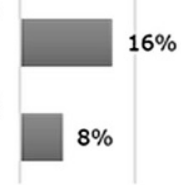

$16 \%$

$0 \%$

$20 \%$

$40 \%$

$60 \%$

$80 \%$

$100 \%$

Abb. 3 Auswirkungen von Covid-19 auf die Arbeitsorganisation

als Reaktion dominieren. Offensichtlich hat Online-Zusammenarbeit zugenommen. Interessant aber auch der Drang nach digitaler Transformation und andere Innovationsthemen (Ebert 2020; Ebert und Duarte 2018; McKinsey 2021). Die Themen hängen mit der Frage aus Abb. 2 zusammen, wobei wir gezielt das Arbeitsverhalten im neuen Normal hinterfragt haben. Hier geht es um wahrgenommene Veränderungen im Lockdown, und nicht um wahrgenommene Herausforderungen. Daher ist „Innovation“ nicht so weit oben, da in der Wahrnehmung die Online-Arbeit als aktuell stärkste Veränderung maßgeblich ist.

\section{Erfolg mit verteilten und virtuellen Teams}

„Zusammenkommen ist ein Beginn. Zusammenbleiben ist ein Fortschritt. Zusammenarbeiten ist ein Erfolg." Henry Ford hat nicht nur die Massenproduktion erfunden und damit das Auto für jedermann erschwinglich gemacht, sondern auch die Team-Arbeit. Verteiltes Arbeiten braucht agile Prozesse mit einem hohen Anteil an Selbststeuerung. Verteilte Agilität bedeutet Zusammenarbeit und gemeinsame Verantwortung für Ergebnisse. Erfolgreiches agiles Arbeiten heißt, dass man den Werkzeugkasten der Methoden kennt und bestmöglich nutzt. Ein täglicher Scrum, also ein kurzer Abstimmungsprozess im gesamte Team mit belastbarer Selbstverpflichtung, bringt mehr als ein komplexes Planungswerkzeug. Da wir ab jetzt konkrete Tipps geben, werden wir Sie als Leser auch immer wieder direkt ansprechen.

Verteilte Teams definieren sich über die Distanz der Menschen im Team Dafür gibt es keine mathematischen Formeln. Distanz ist Wahrnehmung und hat verschiedene Dimensionen: Raum, Zeit und Kultur. Die Erfahrung zeigt, dass Verteilung 


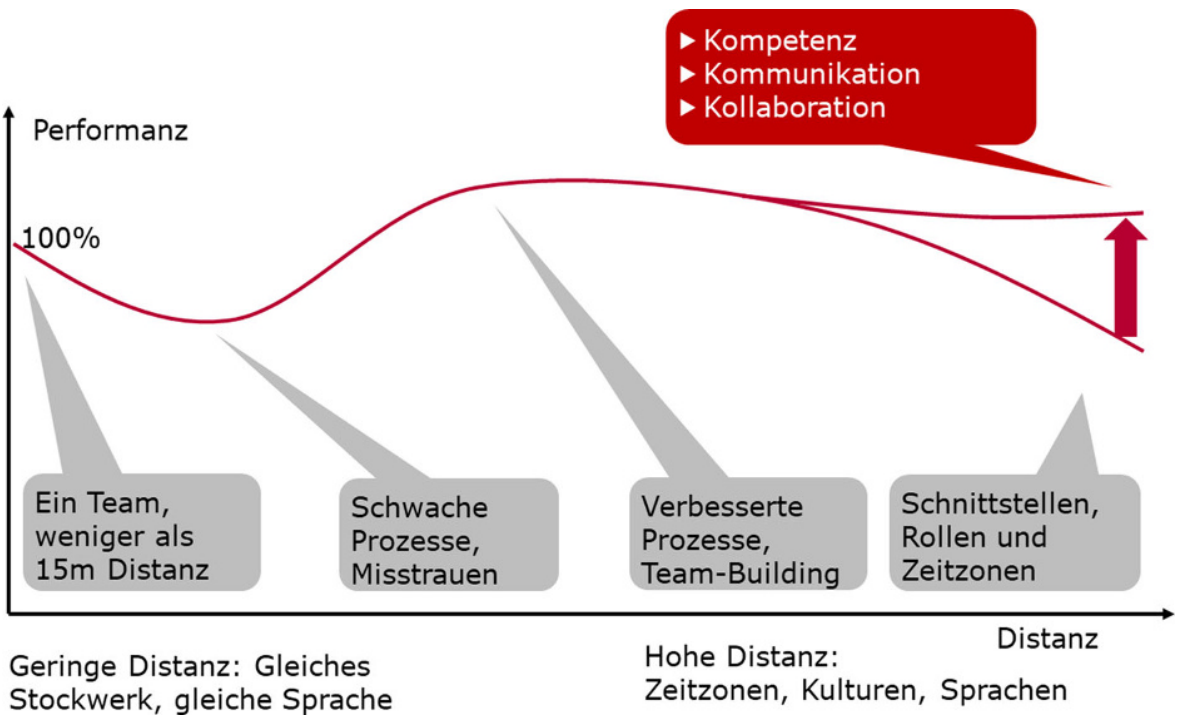

Abb. 4 Performanz und Distanz

früher beginnt, als man wahrhaben will. Es sind nicht Zeitzonen, sondern einige Meter. Ab ungefähr fünfzehn Meter Entfernung schreibt man eher eine Mail, als dass man sich auf den Weg macht, um dann eventuell ein leeres Büro oder den Kollegen am Telefon anzutreffen (Ebert 2020; Schüller und Steffen 2019; Boos et al. 2016).

Distanz beeinflusst Performanz Das muss nicht grundsätzlich eine negative Korrelation sein, wie man es naiverweise annehmen könnte, d.h. größere Distanz reduziert die Produktivität. Oft ist sogar das Gegenteil der Fall, d.h. die Produktivität wächst mit der Distanz - außer die Zeitzonen verhindern jede Kommunikation. Abb. 4 zeigt die Performanz eines Teams abhängig von der Distanz (Ebert 2020). Die Leistungsfähigkeit wird mit wachsender Distanz besser, wenn die Prozesse und Rollen gemeinsam abgestimmt sind. Ein verteiltes agiles Team mit Eigenverantwortung und klaren Aufgaben ist sehr viel produktiver als eine starre Linienorganisation im Großkonzern mit Taylorismus und dadurch wenig motivierten Mitarbeitern. Die drei wesentliche Erfolgsfaktoren für verteiltes Arbeiten sind oben rechts dargestellt. Kompetenz ist offensichtlich am wichtigsten, denn sonst erleben wir Ping-Pong durch Unwissenheit und Fehler. Kommunikation schafft belastbares Vertrauen und Verständnis. Kollaboration schließlich bedeutet ein gutes gemeinsames Verständnis für Prozesse, d.h. Rollen, Verantwortungen, Abhängigkeiten und Aufgaben.

Verteilte Teams verlangen Disziplin - und schaffen mehr Disziplin Dazu braucht es drei Erfolgsfaktoren, die systematisch verankert werden müssen: 

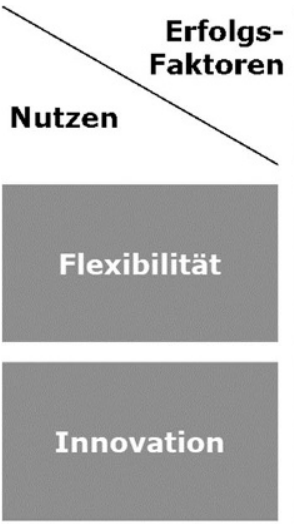

Efizienz
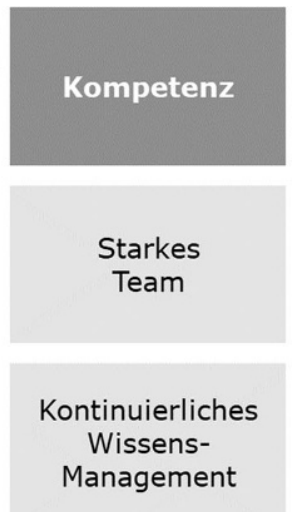

Gute Prozessfähigkeit



Ergebnisorientierte Führung

Klare Aufgaben

Transparente Organisation und Schnittstellen
Kollaboration

Ständig aufgefrischte Soft Skills

Verlässliche Partner

Optimierte IT Infrastruktur

Abb. 5 Balance der Erfolgsfaktoren

- Kompetenz, also harte und weiche Fähigkeiten, Training, Wissensmanagement, Lieferantenmanagement, Lieferantenauswahl, optimale Entwicklungsprozesse, Schnittstellen, Verantwortungen

- Kommunikation, also kulturelles verstehen, aufeinander zugehen, Ressourcenmanagement, betriebswirtschaftliches und technisches Controlling, Prozessüberwachung, Fortschrittskontrolle in Projekten, Management von Dienstqualität oder Service Leve Agreements (SLA)

- Kollaboration, also effiziente Geschäftsprozesse, Teamwork, gemeinsame Ziele und Zielerreichung, Zusammenarbeit über Landesgrenzen und Zeitzonen, effektive Werkzeugunterstützung

Abb. 5 zeigt, wie diese drei Erfolgsfaktoren gemeinsam dazu beitragen, die typischen Ziele Flexibilität, Innovation und Effizienz zu erreichen. Ein starkes Team ist zweifelsohne zwingend nötig und zeigt, wie Flexibilität erreicht wird. Kollaboration mit optimierter IT Infrastruktur schafft Effizienz, da die Mitarbeiter sich häufiger abstimmen, und damit Fehler früher entdeckt werden. Alle neun Kreuzungspunkte gemeinsam sind ein Zeichen für ein nachhaltig erfolgreiches verteiltes Team. Erfolgreiche Arbeit im verteilten Team heißt, dass diese drei Eckpfeiler gleichermaßen gut verankert und verstanden sind.

Wir wollen nun einige Erfahrungswerte aus der Covid-19 Transformation ins neue Normal betrachten, die verteiltes Arbeiten von einem Risiko zu einem Gewinn für ein Unternehmen machen (Ebert 2020, 2014). Die genannten Beispiele sind der Matrix in Abb. 5 entnommen und dort an den Schnittstellen von Erfolgsfaktoren, also Eingangsgrößen, und dem erreichbaren Nutzen, also der Leistungsfähigkeit eines Unternehmens in den eingangs genannten drei Dimensionen von Flexibilität, Innovation und Effizienz (vgl. Abb. 1) dargestellt. 
Kontinuierliches Wissensmanagement In dem Grad, wie sich Mitarbeiter nicht mehr zufällig oder geplant kurzfristig austauschen können, muss sich bei verteiltem Arbeiten das Wissensmanagement massiv verändern. Kontinuierliches Wissensmanagement hat viele Facetten. Beispielsweise führten wir in Regelbesprechungen bei vielen Kunden ein explizites „Knowledge Sharing“ ein. Statt klassischer Scrum wird implizites Wissen explizit herausgearbeitet. Auch die Dokumentation muss sich ändern, denn nicht immer sind alle Mitarbeiter anwesend und haben auch keinen verlässlichen Anker zum Austausch. Die gute Nachricht ist, dass Trainings bereits vorher Online in sehr unterschiedlichen Formaten angeboten wurden, die sofort umsetzbar waren. Webinare, Podcasts, aber auch interaktive Konferenzen ermöglichen den Austausch von Wissen - auch und gerade innerhalb des Unternehmens. Spätestens mit der Corona-Krise hat jeder solche neuen Formate von Online-Wissensvermittlung kennen und schätzen gelernt. Moodle als Online-Lernplattform ist kein Fremdwort mehr. Offensichtlich braucht ein gutes Distanz-Training mehr als nur das Online-Format. Es bedarf völlig neuer Strukturen mit kurzen Modulen, viel Interaktion, und häufigen Abstimmungen mit den verteilten Teilnehmern.

Kontinuierliches Training ist wichtig, um die Teams zu synchronisieren, und um Unklarheiten zu erkennen und sofort auszuräumen. Gutes Training motiviert und fördert Beziehungen und Bindungen der Mitarbeiter - vor allem, wenn sie aus unterschiedlichen Kulturen und Unternehmen kommen. Technische Details müssen intensiv trainiert werden (z. B. Architektur, Bibliotheken, Prozesse, Werkzeuge, Altsysteme). Der Trainingsaufwand muss explizit geplant und budgetiert werden, beispielsweise Zeit und Aufwand, Trainerleistungen. Das gilt gerade für Trainer, also in der Regel erfahrene Mitarbeiter Ihres Hauses, für die dies häufig ein $\mathrm{Zu}-$ satzaufwand bedeutet. Trainingspläne sollten pro Mitarbeiter existieren, um gezielt die jeweiligen Fähigkeiten hochzufahren und vorausschauend anzupassen. Training muss an Diversität und Distanz angepasst werden, wobei sicherlich der persönliche Kontakt zum Trainer am besten ist, obwohl nicht immer realisierbar, wenn der Lieferant sehr schwer zu erreichen und die Fragestellung dringend ist.

Ständig aufgefrischte Soft-Skills In verteilten Teams ist Kommunikation einer der wichtigsten Erfolgsfaktoren. Oftmals wird etwas nicht verstanden, und das jeweilige Kulturmix erlaubt nicht, Zweifel sofort zu klären (Willcocks et al. 2017; Meyer 2016). Führen Sie daher ein verbindliches Kommunikationsprotokoll ein. Stellen Sie eine gemeinsame Projekt-Homepage oder ein Projektportal für alle Projektinformationen, beispielsweise Anforderungen, Data Dictionary, Metriken, Fortschrittsberichte, etc. zur Verfügung. Zur Vereinfachung sollte dieses Portal für alle Ihre Projekte identisch in Struktur und Aussehen sein. Eröffnen und nutzen Sie sowohl regelmäßige als auch spontane Kommunikationskanäle, beispielswese für ein bestimmtes Projekt, für Prozessverbesserungen, für Review-Ergebnisse, etc.

Klare Aufgaben Sie erhalten im verteilten Arbeiten, was Sie vereinbaren und einfordern. Vereinbaren Sie daher konkrete Qualitätsziele pro Arbeitsergebnis bei einem verteilten Projekt. Definieren Sie diese Ziele explizit, z.B. schriftlich als SLA, und messbar (Willcocks et al. 2017; Ebert 2014). Ein SLA ist im verteilten Team kein schwerfälliges Instrument, sondern eine abgestimmte Schnittstellenbe- 
schreibung. Qualität braucht allerdings mehr als nur Abnahmekriterien am Ende. Vereinbaren Sie nachvollziehbare, kaskadierte qualitätssichernde Maßnahmen und Freigabekriterien an den Schnittstellen. Regelmäßige Status-Reviews, Audits und Eingangskontrollen - auf beiden Seiten - sind unabdingbar, um Qualitätsbewusstsein zu erreichen und aufrecht zu erhalten. Vereinbaren Sie daher konkrete qualitätskontrollierende und -sichernde Prozessschritte, um Abnahmeprobleme zu eliminieren, oder aber sie zu eskalieren. Benennen Sie einen Qualitätsmanager, der ggf. projektübergreifend die nötigen Befähigungen aber auch den richtigen Einfluss hat, um rechtzeitig einzugreifen, wenn ein Qualitätsrisiko droht. Legen Sie ein Berichtswesen fest, beispielsweise Templates, das beim Nachvollziehen der qualitätssichernden Maßnahmen hilft.

Ergebnisorientierte Führung Vereinbarungen bringen nichts, wenn dahinter kein Monitoring steckt. Gutes Monitoring der Erreichung von Vereinbarungen ist der Schlüssel für verteiltes Arbeiten. Eine konsequente Verfolgung der (Zwischen-) Ergebnisse ist bei verteilten Projekten wichtig, denn Sie haben keine Möglichkeit, mal nebenbei mit den Beteiligten zu sprechen. Vereinbaren Sie messbare SLAs und halten sie vertraglich fest. Knebeln Sie den Lieferanten nicht primär durch SLAs, sondern durch Beteiligung am wirtschaftlichen Erfolg (oder Misserfolg). Setzen Sie operative Frühwarnindikatoren ein. Diese geben Handlungsspielraum, um rechtzeitig und proaktiv einzugreifen. Erfolg, Projektverfolgung und Schnittstellenmanagement müssen sich an messbaren Kennzahlen orientieren. Dazu gehören Kennzahlen und KPI, beispielsweise Inkremente, Earned Value, Risiken und Linderungsmaßnahmen sowie Qualitätskennzahlen gegenüber vereinbarten Standards und Vorgaben (Ebert 2014).

Optimierte IT-Infrastruktur Verteiltes Arbeiten braucht die passende IT-Infrastruktur. Abgestimmte Werkzeuge sind Pflicht, denn sonst gibt es ständige Reibungsverluste. IT Infrastruktur für Kommunikation, Zugriff auf Dokumente und für das Anforderungs- und Änderungsmanagement ist dabei wesentlich. Dies sind die Fallstricke bei verteilten Teams. Was in der internen Entwicklung gern vernachlässigt wird, beispielsweise versteckte Zusatzkosten oder Nacharbeiten, kommt im verteilten Arbeiten unweigerlich auf den Tisch. Anforderungsänderungen sowie Änderungen im Projektplan sind in der Regel Vertragsänderungen. Häufig werden Änderungen ,stillschweigend vereinbart“, also durch Projektmitarbeiter beider Seiten im laufenden Geschäft, weil die Änderungen kurzfristig nötig sind, dienen aber Ihrem Lieferanten später als Rechtfertigung dafür, Termine und Qualitätsvorgaben nicht einzuhalten.

\section{Führung von Verteilten Teams}

Erfolg im neuen Normal braucht gute Führung. Das zeigt die mittlere Spalte von Abb. 5, und wir wollen hier noch einige konkrete Erfolgsrezepte dazu geben (Ebert 2020). 
Managen verteilter Teams ist die Schlüsselkompetenz Das globale Engineering wird sich zu einer standardisierten Engineering-Management-Methode entwickeln, die von jedem F\&E-Manager beherrscht werden muss. Prozesse und Produktkomponenten werden zunehmend in einem globalen Kontext verwaltet werden. Anbieter aus vielen Ländern werden sich entwickeln, um die Gründung und den Betrieb von globalem Software-Engineering auch für kleine und mittlere Unternehmen in den Hochkostenländern zu erleichtern. Es werden Makler entstehen, die dabei helfen, Partner in verschiedenen Teilen der Welt zu finden und die Offshoring-Kosten zu verwalten (Ebert 2018).

Jeder Mitarbeiter muss seinen Wert ständig neu definieren Wir alle sind ersetzbar und müssen daher ständig neu definieren, wieso jemand in unsere Leistung investieren soll. Die Marke ,ich“ und unser eigener USP wird zum Überlebenskampf. Kein Unternehmen kann heutzutage Geld und Ressourcen in teure Mitarbeiter gerade in Hochlohnländern stecken, ohne dafür einen Return zu erhalten. IT und Softwareentwicklung müssen daher das gesamte Produkt- oder Dienstleistungsgeschäft profitabler machen. Jede Aktivität, jedes Produkt und jedes Projekt müssen die Profitabilität eines Unternehmens messbar erhöhen (Triest und Ahrend 2019). Vereinbaren Sie eine gemeinsame Vision, die in eine Strategie umgesetzt wird, von der operative Ziele abgeleitet werden. Wenn Strategie und Ziele nicht zueinander passen, werden Sie scheitern.

Viele Menschen arbeiten mit angezogener Handbremse Die Motivation fehlt, und wie immer ist die Umgebung daran schuld. Führung bedeutet zuallererst, sich selbst zu führen. Nur wer sich selbst gut führt, kann auch andere führen. Verpflichten Sie sich und das verteilte Team auf gemeinsame Ziele. Feuerwehreinsätze sind normal, sollten aber nicht dazu führen, dass man in operativer Hektik verharrt. Optimieren Sie strategisch. Prüfen Sie im verteilten Team ständig, ob noch alle an Bord sind. Fragen Sie sich und das Team in kurzen täglichen Retrospektiven, wie sie an diesem Tag weitergekommen sind, was sie gelernt haben, und was am nächsten Tag geliefert wird.

In virtuellen Teams kracht es seltener als in traditionellen Teams aber dafür heftiger Der Grund ist offensichtlich. Man plätschert online durch die Regeltermine. Keiner mag persönliche Gefühle darstellen und kehrt Probleme unter den Teppich. Doch irgendwann kommt der Zeitpunkt, wenn unter dem Teppich kein Platz mehr ist. Termine werden verschoben, die Qualität stimmt nicht, es wird E-Mail PingPong gespielt. Dann kommen plötzlich die Historien von Konflikten auf den Tisch, die hätten längst gelöst werden müssen.

Nicht jeder Mitarbeiter ist es gewohnt, sich selbst zu organisieren Die Zahl der Ablenkungen wächst mit der Distanz des Teams. Das können banale technische Themen ein, die uns über Maß beschäftigen. Oder auch die Hausarbeit, die uns ablenkt. Helfen Sie Ihren Mitarbeitern auf dem Weg, sich selbst zu organisieren. Bereiten Sie gemeinsam eine Aufgabenliste mit Aufwandschätzung und Prioritäten der einzelnen Aufgaben vor. Das lohnt sich immer, denn die Mitarbeiter lernen 
gute Arbeitsgewohnheiten, auf die Sie in komplexen Situationen aufbauen können. Punkten Sie als Führungskraft, indem Sie Wertschätzung und Verständnis zeigen. Das schafft das nötige Vertrauen, und Ihre Mitarbeiter werden es Sie umgekehrt ebenso spüren lassen.

Vorhandene Schwächen werden im verteilten Arbeiten multipliziert Es fehlt die frühe Sichtbarkeit, und man hat weniger Chancen für informelle Abstimmungen, die im traditionellen Team Druck abbauen und offenen Punkte - auch zwischenmenschlich - auflösen. Beispiel: Wer mit Kollegen direkt kommunizieren kann, braucht weniger Details und Dokumente. Man kann ja schnell mal zum Kollegen. Das geht im verteilten Team nicht. Dort spricht man weniger miteinander, und macht daher Fehler, wo Dinge unscharf beschrieben sind. Versäumte Fristen, schlechte Ergebnisse und zwischenmenschliche Konflikte sind die Folge. Was für virtuelle Teamleiter anders ist, ist der unpersönliche Kontext, in dem diese Leistungsprobleme stattfinden. Dokumentieren Sie gemeinsame Erwartungen, einigen Sie sich auf Verantwortlichkeitsprozesse, geben Sie regelmäßiges Feedback, verfolgen Sie einen expliziten Verbesserungsplan, in den Sie regelmäßig mit dem Team schauen.

Führungskräfte müssen die Gefühle ihrer Mitarbeiter antizipieren und verstehen Führen Sie dazu den Perspektivwechsel durch und versetzen Sie sich direkt in die andere Person: Was könnten die anderen fühlen? Welche Empfindungen könnte eine Vorgabe auslösen - selbst, wenn diese gar nicht intendiert ist? Gehen Sie als Führungskräfte zunächst immer erst von der ungünstigen und negativen Interpretation Ihrer Kommunikation aus. Viele Menschen sehen in Führungskräften potenzielle Feinde. Das mag archaisch sein und passt auch gar nicht zum partnerschaftlichen Umgang miteinander. Daher braucht verteilte Kommunikation mehr Erklärungen und Transparenz, als das in einem klassischen Büro mit Kaffeeküche gewohnt war. Umgekehrt ist es von Vorteil für alle, die verteilte Teams und Projekte geführt haben. Denen kann auch in ,engeren“ Situationen mit Vor-Ort-Präsenz nichts passieren.

Seien Sie sensibel bei allen Auffälligkeiten Wenn jemand im Zeitverzug mit Aufgaben ist, ist das aus Sicht der Arbeitsergebnisse nicht sofort erkennbar. Fassen Sie auch mal telefonisch nach. Achten Sie auf auffälliges Verhalten. Er ist bei einem Teamgespräch untypisch ruhig, er erhöht oder verringert die Häufigkeit, mit der er kommuniziert, oder er scheint besonders ängstlich, frustriert oder geistig abwesend. Achten Sie auf Interaktionen im Team, auf Mails und Telefonate. Sind Mitarbeiter vor einem wichtigen Termin seltsam entspannt? Buchen sie zu wenig Aufwand, wenn Kollegen überlastet sind? Zögern Sie nicht, wenn solche Auffälligkeiten auftreten. Greifen Sie zum Telefonhörer. Die Führung eines in Schwierigkeiten geratenen Teammitglieds ist ein kritischer Prozess, der viel Zeit, Aufmerksamkeit und Einfühlungsvermögen erfordert - am besten von Angesicht zu Angesicht, denn die physische Nähe stärkt die Beziehung. All das lässt sich auf Distanz nicht so leicht erkennen. Empathie auf Distanz braucht besondere Erfahrung.

Zwischenmenschliche Konflikte sollten Sie persönlich ausgleichen Das braucht keine Reise. Häufig genügt ein einfühlsames Gespräch in einer Online Video-Kon- 
ferenz. Mediation klappt auch virtuell, entweder durch Sie, einen anderen Vorgesetzten oder jemanden mit Konfliktlösungserfahrung. Natürlich ist das persönliche Gespräch ideal, oder Sie können auch mit Video gute Ergebnisse erreichen. Legen Sie dazu klare Regeln für den Gesprächsablauf fest. Ohne Blickkontakt am Telefon werden sich die Teilnehmer wahrscheinlich gegenseitig unterbrechen. Nutzen Sie daher ein Video-Gespräch, wo Sie mindestens Mimik erkennen, besser noch die gesamte Körpersprache.

Führung eines virtuellen Teams ist immer auch ein Experimentieren Man kann vorher nicht alles im Sandkasten üben. Jede neue Herausforderung reichert Ihren Problemlösungsbaukasten an, wenn Sie von Ihren entfernten Kollegen lernen. Mit den richtigen Werkzeugen und den richtigen Mentoren werden Sie die Vorteile eines engen Teams ernten, egal, wie weit die Mitglieder physisch entfernt sind.

\section{Check: Ihr Start in verteiltes Arbeiten}

Oft stellt sich die Frage nach den Minimalvoraussetzungen für verteilte Teams und virtuelle Projekte. Den Betroffenen ist klar, dass sie sich ändern müssen, und auch die Prozesse professionaler werden müssen. Aber was ist wirklich wichtig, und in welcher Reihenfolge?

Daraus ist die folgende Checkliste entstanden. Sie ist ein Impulsgeber und Augenöffner. Sie zeigt Stärken und Schwächen. Vor allem deutet sie auf „blinde Flecke“, die man noch nicht ausreichend bedacht hat. Hier diese minimalistische Checkliste, die auf die wesentlichen Randbedingungen eingeht. Beantworten Sie die folgenden 15 Fragen spontan mit „Ja“ oder „Nein“:

- Ist Ihr Auftrag für das verteilte Arbeiten klar?

- Passen die Aufgaben der virtuellen Zusammenarbeit für Sie?

- Gibt es in der Aufgabenbeschreibung genug stimulierende Inhalte?

- Kommen Sie mit dem Team und der Führung klar?

- Sind Sie im Grund positiv denkend und argumentieren konstruktiv?

- Arbeiten Sie gerne auf Distanz und ohne direkten Kontakt?

- Haben Sie in den virtuellen Teams explizite Werte und Regeln aufgebaut?

- Werden diese Regeln eingehalten?

- Kommunizieren Sie bewusst auf verschiedenen Kanälen?

- Arbeiten die relevanten Funktionen im Unternehmen miteinander für gemeinsame Ziele?

- Führen Sie (auch indirekt ohne explizite Führungsverantwortung) ergebnisorientiert?

- Verfolgen Sie im Team Status, Aufgaben, Vereinbarungen und Risiken zumindest wöchentlich?

- Haben Sie Standards für Prozesse und IT-Infrastruktur, die eingehalten werden?

- Geben Sie Ihr Knowhow auch an ihnen unbekannte Mitarbeitende weiter?

- Würden Ihre Kollegen im virtuellen Team die gleichen Antworten geben? 
Diese Checkliste sollte im verteilten Team periodisch genutzt werden, um bei jedem Durchlauf einige Schwachstellen auszuwählen und die nötige Verbesserung zu starten. Sie wird typischerweise von jedem Team-Mitglied ausgefüllt, was in der Summe ungefähr fünf Minuten braucht. Optional lässt sich noch eine TrendFrage hinzufügen, beispielsweise: ,Welche Veränderung in der vergangenen Periode hatte den größten Einfluss?“ Das lässt sich zusätzlich differenzieren nach positiv vs. negativ.

Was machen Sie mit dem Resultat der Checkliste? Bewerten Sie zunächst Ihr Ergebnis anhand der Antworten. Ja=1 Punkt, Keine Ahnung=0 Punkte, Nein $=-1$ Punkt. Zählen Sie nun alle Punkte zusammen.

- 12-15 Punkte: Gut. Qualität ist kein Status Quo, sondern muss ständig neu erkämpft werden. Verbessern Sie sich kontinuierlich anhand der Projektergebnisse und Benchmarks mit Mitbewerbern und Klassenbesten.

- 8-11 Punkte: Lernen. Sie sind auf dem richtigen Weg, aber es braucht noch substanzielle Anstrengung. Priorisieren Sie anhand der endeckten Schwachstellen.

- <8 Punkte: Lücken schließen. Die entdeckten Schwachpunkte dringend verbessern. Dieser Beitrag hilft Ihnen mit Lösungen und Impulsen zur Verbesserung.

Adressieren Sie die Schwachstellen mit Lösungen für verteiltes Arbeiten, die sich in der Praxis bewährt haben (Ebert 2020; Schüller und Steffen 2019; Boos et al. 2016; Triest und Ahrend 2019). Beispielsweise steht in der Checkliste die Frage „Führen Sie (auch indirekt ohne explizite Führungsverantwortung) ergebnisorientiert?“ Offensichtlich geht es hier um die persönliche Wahrnehmung. Je nach Naturell werden einige Mitarbeiter euphorisch reagieren, vielleicht auch, weil sie endlich schalten und walten können, wie sie meinen, dass es gut ist. Eine Mehrheit wird zurückhaltend antworten, wohlwissend um die Risiken eigener Arbeitsorganisation. Als Führungskraft befrieden Sie die Ängste durch klare Ansagen und Verantwortungen sowie häufige virtuelle Team-Besprechungen. Sprechen Sie mit Fallbeispielen und guten Impulsen im Team-Meeting offensiv zu diesen Risiken. Bestimmt gibt es weitere gute Ideen. Häufig sind die Mitarbeiter zurückhaltend im Scrum. Stimulieren Sie klare Aussage. Fragen Sie gerade in virtuellen Konstellationen mit wechselnder Reihenfolge alle Mitarbeiter im Scrum ab. Immer wieder erleuchtend, dass plötzlich jene Personen den Mund aufmachen, die sonst im Trubel der Lautsprecher und Besserwisser untergehen.

\section{Fazit: Das neue Normal ist ständige Veränderung}

„Jeder war Zeuge dieser ungeheuren Verwandlungen, jeder war genötigt, Zeuge zu sein. Für unsere Generation gab es kein Entweichen, kein Sich-abseits-Stellen wie in den früheren." Stefan Zweig hat unsere aktuelle Epoche unwissentlich vorweggenommen (Zweig 1942). Selten hat sich die Menschheit in solch kurzer Zeit so schnell verändert wie seit dem Frühjahr 2020. Ständige Veränderung mit hoher Geschwindigkeit war bisher das Zeichen für innovative Start-Ups. Mit unserer Industrie-Umfrage und dieser Studie haben wir nun gelernt, dass stetige Innovation 
uns alle betrifft. Wir alle sind in ständiger Veränderung, sei es im Unternehmen mit Produkten und Geschäftsmodellen, oder sei es persönlich mit Kompetenzen und Verhaltensweisen.

Mit der Covid-19-Pandemie haben sich unsere Arbeit, unsere Gesellschaft und unser Leben auf einen Schlag radikal geändert (Ebert 2020; Stelter 2020). Althergebrachte Paradigmen, wie Team-Arbeit, Konferenztische, Hinterzimmer und Reisen waren vergessen. Verteiltes Arbeiten war plötzlich möglich, denn es wurde zur Pflicht. Weltweit. Man arbeitet verteilt - und es funktioniert. Veränderungsprozesse sind in kurzer Zeit zu einem Standardwerkzeug in vielen Unternehmen geworden. Virtuelle Meetings und mobiles Arbeiten, früher eher die Ausnahme und ständig aufgeschoben, wurden in kurzer Zeit weltweit realisiert und für praxistauglich befunden.

Innovation wird kaum am eigenen Schreibtisch im so genannten stillen Kämmerchen erreicht. Innovation braucht Austausch, mit Andersdenkenden und über die eigene Erfahrung und Kultur hinaus. Daher ist as aktuelle Einschließen in die eigene Blase auf der eigenen Coach auch so gefährlich. Wer sich nicht bewegt, wird abgehängt, und das ist der wirkliche „Digital Divide“ unserer Gesellschaft. Kollaboration und verteiltes Arbeiten definieren unsere Zukunft als wesentliche Veränderung. Die Geschichte hat immer wieder gezeigt, dass die Vermischung der Gene das Beste ist, was auf dem Weg der Evolution getan werden kann.

Die Welt der Coronomics braucht Nähe in der Distanz. Verbundenheit hat sich neugestaltet und ist im besten Fall hybrid mit klassischer Begegnung und parallelem Online Format. Begegnung wird neu definiert mit einer im wahrsten Sinn des Wortes greifbaren holografischen virtuellen Realität. Lieferwege und Lieferanten, Autonomie und Abhängigkeit, Öffnung und Schließung von Märkten werden neu ausbalanciert.

Unser Leben wird mit ständiger Veränderung komplexer, denn es gibt in diesem evolutionären Prozess keine vorher gedachten Antworten. Zukunftsfähig ist das, was allfällige Unwägbarkeiten und Risiken auf einer neuen Ebene verbindet. Oder mit den Worten von Charles Darwin, der einer der ersten wirklich global agierenden Wissenschaftler war: „Es sind nicht die Stärksten der Spezies, die überleben, oder die Intelligentesten, sondern diejenigen, die am besten auf Veränderungen reagieren." Wer langfristig wettbewerbsfähig bleiben will, muss auf die Herausforderungen schnell und flexibel reagieren. Nutzen auch Sie jede Krise als Chance und gestalten Sie Ihre Zukunft und die Ihres Unternehmens ständig neu.

\section{Literatur}

Boos M, Hardwig T, Riethmüller M (2016) Führung und Zusammenarbeit in verteilten Teams. Hogrefe, Göttingen

Ebert C (2014) Risikomanagement kompakt: Risiken und Unsicherheiten bewerten und beherrschen, 2. Aufl. Springer, Heidelberg

Ebert C (2020) Verteiltes Arbeiten, 2. Aufl. Springer, Wiesbaden

Ebert C, Duarte CHC (2018) Digital Transformation. IEEE Softw 35(4):16-21

McKinsey (2021) The future of work after COVID-19. Special Report. https://www.mckinsey.com/ featured-insights/future-of-work/the-future-of-work-after-covid-19. Zugegriffen: 26. Febr. 2021

Meyer E (2016) The culture map: decoding how people think, lead, and get things done across cultures. Public Affairs, New York 
Schüller A, Steffen A (2019) Die Orbit-Organisation: In 9 Schritten zum Unternehmensmodell für die digitale Zukunft, 2. Aufl. Gabal, Offenbach

Stelter D (2020) Coronomics. Campus Verlag, Frankfurt

Triest S, Ahrend J (2019) Agile Führung. Mitarbeiter und Teams erfolgreich führen und coachen. Mitp, Frechen

Willcocks LP, Lacitity MC, Sauer C (2017) Outsourcing and offshoring business services. Palgrave Macmillan, London

Zweig, S (1942) Erinnerungen eines Europäers. Hamish-Hamilton, London

Christof Ebert ist Geschäftsführer bei Vector. Er unterstützt Kunden bei Produktstrategie, Entwicklung und agiler Transformation und arbeitet in verschiedenen Aufsichtsgremien von Unternehmen. Zuvor war er zwölf Jahre bei einem IT Konzern in weltweiten Führungsaufgaben. Als Business Angel und Professor an der Universität Stuttgart und der Sorbonne in Paris stimuliert er Innovationen. Er ist in den HerausgeberKomitees von Zeitschriften wie IEEE Software, Software Quality Journal und dem Journal of Systems and Software. Als Musiker spielt er Klavier, Synthesizer und Kirchenorgel. 